\title{
Central Nervous System Vasculitis Due to Tuberculous Meningitis in A 23-year-old Adult with Pharmacological Immunosuppression
}

\section{Francisco Javier Rivas-Ruvalcaba, Melissa Hernández-Vega, Arturo González-Lara and Ildefonso Rodríguez-Leyva*}

Neurology Service, Hospital Central "Dr. Ignacio Morones Prieto" and Facultad de Medicina, Universidad Autónoma de San Luis Potosí, Venustiano Carranza, Los Filtros, San Luis Potosí, San Luis Potosí, México

*Corresponding Author: Ildefonso Rodríguez-Leyva, Head Neurology Service, Hospital Central "Dr. Ignacio Morones Prieto" and Facultad de Medicina, Universidad Autónoma de San Luis Potosí, Venustiano Carranza, Los Filtros, San Luis Potosí, San Luis Potosí, México.
Received: November 05, 2021

Published: November 24, 2021

(C) All rights are reserved by Ildefonso

Rodríguez-Leyva., et al.

\begin{abstract}
We present a 23-year-old man with a history of ankylosing spondylitis treated with methotrexate and sulfasalazine presented to the emergency room with a headache and a history of night sweats and weight loss. On a physical examination, he was drowsy, had dysmetria on his left hand and positive meningeal signs. In addition, the lumbar puncture had a positive PCR for Mycobacterium tuberculosis, so he was managed with rifampin, ethambutol, pyrazinamide, isoniazid and dexamethasone and was discharged.

After the discharge, he was readmitted because he experienced acute dysarthria, and an MRI showed evidence of an ischemic stroke with findings suggestive of central nervous system vasculitis. After initiation of secondary prophylaxis and high dose steroid, he was discharged and could stand up by himself and had partial recovery of dysarthria.
\end{abstract}

Keywords: Pyrazinamide; Tuberculous Meningitis; Immunosuppression

\section{Antecedents}

Mycobacterium tuberculosis-related infections are a significant public health issue because of the great morbidity that causes and a broad spectrum of diseases that can lead to a great variety of clinical presentations.

The World Health Organization estimates that 1 of every four persons has a latent infection of the bacilli. However, not everyone develops the disease. In 2019, there were 10 million new cases of tuberculosis [1]. Although evidence of a decrease in the incidence of the disease has been reported, in undeveloped countries, this is a widespread problem and represents a public health issue.

\section{Case Presentation}

A 23-year-old man was referred to the neurology service because of a history of headache, weight loss, fever and night sweats.
In addition, he had a family history of ankylosing spondylitis on his father. Three weeks before his admission, he complained of low back pain and headache. He was seen by a rheumatologist who suspected ankylosing spondylitis because of his family history and the clinical manifestations. So, he started treatment with methotrexate $2.5 \mathrm{mg}$ on Monday, Wednesday and Friday, Sulfasalazine $500 \mathrm{mg}$ Three times a day; also, he had antecedent of arterial hypertension, managed with losartan $50 \mathrm{mg}$ twice a day and amlodipine $5 \mathrm{mg}$ once a day.

He presented in the emergency room for a picture of three weeks of holocranial headache, worsening in the next few hours. We found the patient drowsy and with inattention. He had dysmetria on the left hand, nuchal rigidity, Binda sign was positive, and we found thunder jolt accentuation of headache; the rest of the physical examination was irrelevant. 
The clinical diagnosis was subacute meningitis; because of clinical signs and history of pharmacologic immunosuppression, we had a cranial MRI, which revealed granulomatous lesions on the left orbitofrontal lobe, a hyperintense image on the rostral medulla and basal arachnoiditis (Figure 1A, Figure 1B and Figure 2A). A lumbar puncture was performed, the opening pressure was $7 \mathrm{~cm} \mathrm{H} 0$, the cerebrospinal fluid was clear, and the cell count was 48 cells with $100 \%$ of polymorphonuclear cells and $0 \%$ of monocytes, the protein was $280 \mathrm{mg} / \mathrm{dL}$ and glucose of $19 \mathrm{mg} / \mathrm{dL}$, a direct Gram stain was negative, and the polymerase chain reaction for Mycobacterium Tuberculosis was positive. HIV tests were negative, and the rest of the blood tests were non-relevant. We started treatment with rifampin, ethambutol, pyrazinamide, isoniazid and dexamethasone. The dysmetria, headache and confusion improved after eight days of inpatient management, so the patient was discharged home.

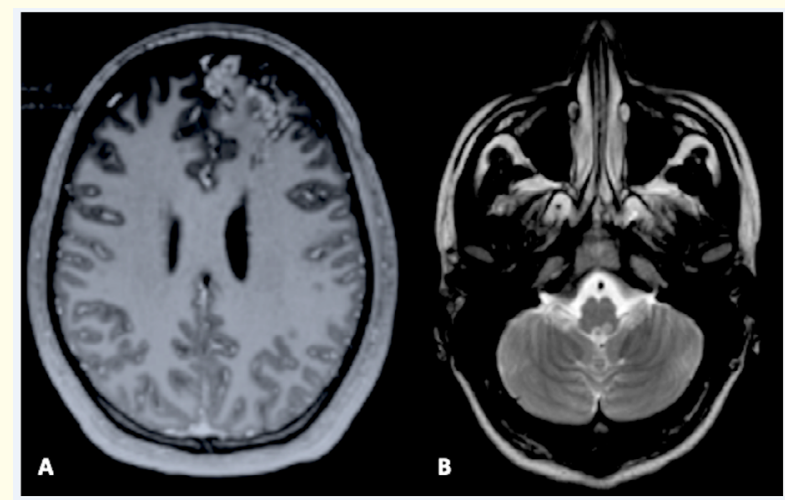

Figure 1: A. Axial FLAIR-weighted MRI showed granulomatous lesions on the left orbitofrontal lobe. B. Axial T2-weighted MRI Showing a hyperintensity on the posteromedial aspect of the rostral medulla.

Twelve days after the discharge, the patient had an event of sudden dysarthria, followed by confusion, so it was admitted again with the suspicion of an ischemic stroke due to a vasculitis secondary to tuberculous meningitis. A second MRI was performed, which revealed an ischemic stroke on the anterior limb of the left internal capsule (Figure 2B), and the magnetic resonance angiography showed narrowing of multiple vessels (Figure 3). After three days of the event, the patient was discharged with dysarthria and generalized debility with 4 points in the modified Rankin scale.

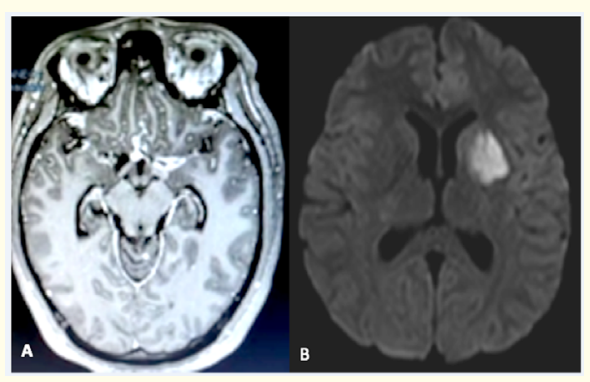

Figure 2: A. Axial T1 gadolinium-enhanced, which reveals basal arachnoiditis. B. Axial Diffusion-weighted sequence with an ischemic lesion.

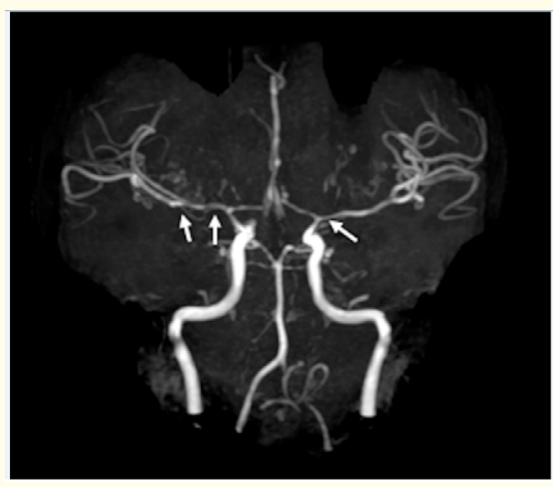

Figure 3: Magnetic resonance angiography with narrowing on the M1 portion of both medial cerebral arteries (arrows).

\section{Investigations}

We took a diagnostic serum test was taken at the emergency room. The complete blood count revealed normal haemoglobin levels, a total of leucocytes of 12,300 cell/dL, the differential was 9,200 neutrophils, 1800 lymphocytes and 1,100 monocytes, the platelet count was 213,000 . In addition, the glucose, creatinine, urea and serum electrolytes were average.

We practiced a lumbar puncture at admission with an opening pressure: $7 \mathrm{~cm} \mathrm{H2O}$; it was a xanthochromic fluid with a Cell count 50 cell/mm3: 100\% polymorphonuclear, 0\% monocytes, protein: $280 \mathrm{mg} / \mathrm{dL}$, glucose $34 \mathrm{mg} / \mathrm{dL}$; Gram stain: negative, India ink test: negative and a polymerase chain reaction for Mycobacterium tuberculosis con cerebrospinal fluid were positive. 
The serology for HIV, B hepatitis virus and C hepatitis virus was negative.

A chest X-ray was practised and was irrelevant, without pulmonary tuberculosis, although a serial sputum Ziehl-Nielsen stain was negative of tuberculosis.

\section{Differential diagnosis}

The initial diagnostic consideration was acute bacterial meningitis. This logical possibility comes to mind when a patient with a history of headache and fever is presented in the emergency room. Commonly, patients have an acute course of days, and they have the triad of headache, fever and neck stiffness [2]. Among the risk factors associated with acute bacterial meningitis are the absence of vaccination against streptococcus pneumoniae, type B Haemophilus influenzae, age under five years old or above 65 years. For Listeria monocytogenes, age, immunosuppression and history of alcohol consumption are typical risk factors [3].

This patient had a history of three weeks of headache and a recent diagnosis of ankylosing spondylitis, for which he was taking immunosuppressant agents.

In this patient, the subacute-chronic course of symptoms was not suggestive of acute bacterial meningitis. The lumbar puncture had a negative Gram stain. In addition, the cerebrospinal fluid culture did not find bacterial growth.

Another diagnosis we considered was meningeal cryptococcosis. Immunosuppressed patients are at higher risk of having a cryptococcal infection. Cryptococcus neoformans is one of the most common pathogens causing meningitis among patients with immunosuppression. It is an invasive fungal infection of encapsulated yeasts of cryptococcus neoformans and similarly to cryptococcus gattii [4]. Besides immunosuppression, the most relevant risk factor is exposure to soil, bird droppings, and it has recently been found that eucalyptus trees could be a source of this infection [5].

Cryptococcal meningitis has been a significant cause of morbidity and mortality among patients with this infection. The clinical course is meningitis with a severe headache due to elevated intracranial pressure and could be accompanied by cranial neuropathies [6]. However, this patient's lumbar puncture was not compatible with cryptococcal meningitis because of a negative India ink test, and the biochemical characteristics of the cerebrospinal fluid did not support this diagnosis.

The lumbar puncture was compatible with tuberculous meningitis, and the clinical course of weeks with headache, fever and immunosuppression, we decided to perform a mycobacterium tuberculosis PCR on CSF, and this was positive.

The patient returned for medical attention because of acute dysarthria, and the MRI showed an ischemic stroke and lesions that suggested central nervous system vasculitis. Central nervous system (CNS) vasculitis related to Mycobacterium tuberculosis infection is rare; children and individuals infected with human immunodeficiency virus (HIV) are at the highest risk. Stroke can occur in up to $15-60 \%$ of the patients with CNS vasculitis due to tuberculosis. The manifestations of tuberculous meningitis can mask the presentation; some patients' stroke is clinically silent or can be insidious in expression [7].

\section{Treatment}

The patient was given rifampin, isoniazid, pyrazinamide and ethambutol (150 mg, $75 \mathrm{mg}, 400 \mathrm{mg} 300 \mathrm{mg}$, respectively) and dexamethasone $(0.4 \mathrm{mg} / \mathrm{kg} /$ day $)$ and pyridoxine supplementation with $50 \mathrm{mg}$ daily because of the evidence of tuberculous meningitis, and we started oral ondansetron for managing nausea and vomiting.

Also, because of the evidence in MRI of ischemic lesions in areas of granulomatous reaction, we added aspirin $100 \mathrm{mg}$ daily as a treatment for a central nervous system vasculitis secondary to tuberculous meningitis.

\section{Outcome and follow-up}

We followed the patient by three weeks after the second discharge, and he was improving the dysarthria, and he was able to stand up by himself, with 2 points on the modified Rankin scale.

However, he was still complaining of nausea and vomiting; despite ondansetron, we believe this is a complication due to the lesion on the rostral medulla.

\section{Discussion and Conclusion}

This patient had evidence of CNS involvement due to M. Tuberculosis, as the PCR of the CSF confirmed the diagnosis. According 
to the NICE Guidelines for Tuberculosis [8], we first managed the infection with the four main antibiotics and pyridoxine supplementation. Also, we added dexamethasone with a dose of $0.4 \mathrm{mg} / \mathrm{kg} /$ day because it has been proved that it reduces mortality [9].

In the second hospitalization, we managed the patient as an ischemic stroke. An initial diagnostic workup for common causes was negative, so we repeated the MRI and found suggestive data of CNS vasculitis, which were associated with tuberculous meningitis.

It has been reported that TB-related infarcts tend to occur in the so-called "TB zone" [10]. There are different pathways through which an infection can injure blood vessels. For example, the pathogen may infect the endothelium or just bind to the endothelial surface, and it can trigger an immune or a toxic response against the endothelium. In the central nervous system context, tuberculosis infection and vasculitis are usually the rules, and the vessel walls are invaded by mononuclear cells, mainly affecting the adventitia and the media [7]. Focal deficits in patients with tuberculous meningitis can occur in up to $10-47 \%$ of the patients according to the different series, with the highest incidence accounting from a Taiwanese cohort. Neurological deficits frequently involve deep structures (basal ganglia and subcortical structures); thus, symptoms like aphasia, apraxia's, and agnosia are less likely to occur. Most infarcts are located in the Tuberculous zone, which arises in an area supplied by the branches of the lenticulostriate arteries and thalamic-perforating branches of the MCA [11].

For this patient, we managed the stroke with secondary prevention. In addition, we extended the time using steroids as an empiric treatment due to previous experience with CNS vasculitis related to Tuberculous meningitis. In those cases, we found frequent readmissions and poor prognoses on patients with a short course of steroids.

\section{Learning Points/Take Home Messages}

- Meningitis could be a presentation of tuberculosis, even in patients without pulmonary disease.

- Immunocompromised patients are at greater risk of having tuberculous meningitis.

- Lesions on the area postrema have a poor response to antiemetic therapies.

- Central nervous system vasculitis secondary to tuberculous meningitis is a common complication.
- $\quad$ Steroid therapy and aspirin improve the clinical outcome and diminish the probability of new strokes in patients with central nervous system vasculitis secondary to tuberculous meningitis.

\section{Bibliography}

1. World Health Organization - tuberculosis.

2. van de Beek D., et al. "Community-acquired bacterial meningitis”. Nature Reviews Disease Primers2 (2016): 16074.

3. McGill F., et al. "Acute bacterial meningitis in adults". Lancet 388.10063 (2016): 3036-3047.

4. Mada PK., et al. "Cryptococcus". En: Stat Pearls. Treasure Island (FL): Stat Pearls Publishing (2021).

5. Elhariri M., et al. "Eucalyptus Tree: A Potential Source of Cryptococcus neoformans in Egyptian Environment”. International Journal of Microbiology 2016 (2016): 4080725.

6. Sloan DJ and Parris V. "Cryptococcal meningitis: epidemiology and therapeutic options". Clinical Epidemiology 6 (2014): 169182.

7. Younger DS and Coyle PK. "Central Nervous System Vasculitis due to infection”. Neurology Clinic 37.2 (2019): 441-463.

8. Recommendations | tuberculosis | guidance | NICE (2021).

9. Prasad K., et al. "Corticosteroids for managing tuberculous meningitis". Cochrane Database of Systematic Reviews 4 (2016): CD002244.

10. Tai M-LS., et al. "Cerebral infarction pattern in tuberculous meningitis". Scientific Reports 6.1 (2016).

11. Katrak SM. "Vasculitis and stroke due to tuberculosis". In: Caplan LR, Bogousslavsky J, editors. Uncommon Causes of Stroke. 2nd ed. Cambridge: Cambridge University Press (2008): 4146.

\section{Volume 4 Issue 12 December 2021}

(C) All rights are reserved by Ildefonso Rodríguez-Leyva., et al. 